\title{
Intense vibronic modulation of the chiral photoelectron angular distribution generated by photoionization of limonene enantiomers with circularly polarized synchrotron radiation
}

Mohammad M. Rafiee Fanood ${ }^{1,+}$, Hassan Ganjitabar ${ }^{2}$, Gustavo A. Garcia ${ }^{3}$, Laurent Nahon ${ }^{3}$, Stefano Turchini ${ }^{4}$, Ivan Powis ${ }^{2, *}$

\footnotetext{
${ }^{1}$ LaserLaB Amsterdam, VU University Amsterdam, De Boelelaan 1081, 1081 HV Amsterdam, The Netherlands.

${ }^{2}$ School of Chemistry, The University of Nottingham, University Park, Nottingham NG7 2RD, UK.

${ }^{3}$ Synchrotron SOLEIL, I'Orme des Merisiers, Saint Aubin BP 48, 91192 Gif sur Yvette Cedex, France.

${ }^{4}$ Istituto Struttura della Materia-CNR (ISM-CNR) Via del Fosso del Cavaliere 100, 00133 Roma, Italy.

† Current address: Department of Imaging Physics, Delft University of Technology, Lorentzweg 1, 2628 CJ Delft, The Netherlands
}

*Corresponding Author:

Tel: $\quad$ +441159513467

E-mail: ivan.powis@nottingham.ac.uk 


\section{Abstract}

Photoionization of the chiral monoterpene limonene has been investigated using polarized synchrotron radiation between the adiabatic ionization threshold, $8.505 \mathrm{eV}$, and $23.5 \mathrm{eV}$. A rich vibrational structure is seen in the threshold photoelectron spectrum and is interpreted using a variety of computational methods. The corresponding photoelectron circular dichroism-measured in the photoelectron angular distribution as a forward-backward asymmetry with respect to the photon direction-was found to be strongly dependent on the vibronic structure appearing in the photoelectron spectra, with the observed asymmetry even switching direction in between the major vibrational peaks. This effect can be ultimately attributed to the sensitivity of this dichroism to small phase shifts between adjacent partial waves of the outgoing photoelectron. These observations have implications for potential applications of this chiroptical technique, where the enantioselective analysis of monoterpene components is of particular interest.

\section{Introduction}

Recent high resolution studies of photoelectron circular dichroism (PECD) in substituted chiral oxirane molecules have revealed an unexpectedly strong vibrational dependence..$^{[1-3]}$ In extremis the associated chiral asymmetry in the forward and backward photoemission may switch $-6 \% \leftrightarrow+6 \%$ on the excitation of a specific vibrational mode. To the uninitiated the magnitude of this asymmetry will itself be a surprise, exceeding more traditional chiroptical asymmetries by orders of magnitude. But even more surprising is the complete reversal of the forward-backward electron scattering propensities that this asymmetry indicates, consequent upon the simple excitation of a weak vibration that is barely perceptible in the regular photoelectron spectra. More generally, the asymmetry detected by the PECD technique displays much more dramatic vibrational dependence for these oxiranes than could be anticipated from other photoionization measurements. An explanation can be found in the enhanced sensitivity to electron scattering phase, hence to both static and dynamic aspects of the molecular framework (nuclei), that is inherent in the chiral dipole matrix elements probed by PECD. ${ }^{[3,4]}$ There is therefore no reason to expect that these pronounced vibrational effects are confined to the small oxiranes, and here we seek to establish whether similar dependencies can be detected in a very different class of chiral molecule.

The monoterpene isomers, $\mathrm{C}_{10} \mathrm{H}_{16}$, are prominent amongst biogenic emissions of volatile compounds (BVOCs), and of these limonene is arguably the most frequently encountered. While clearly associated with citrus fruits, limonene is emitted from many plant species, including forestation ${ }^{[5]}$ and even the marine ecosphere.${ }^{[6]}$ Limonene is one of the most frequently cited examples of a chiral molecule whose perceived odour depends upon the specific enantiomer sampled, and the varying enantiomeric distribution of biogenic limonene with plant type and environment is emerging to be of significant interest ${ }^{[5,7]}$ In the modern urban environment also, enantiomeric limonene is becoming ubiquitous ${ }^{[8,9]}$ due to its nearly universal use as a fragrance in toiletries, air fresheners, and cleaning products.

The widespread incidence of limonene emissions, and especially an increased appreciation of the significance that may be attributed to its enantiomeric ratio, create a need for convenient, chirally sensitive analysis of limonene vapours. In a recent study we showed how enantioselective identification of limonene vapour in a multicomponent mixture could be achieved by using massselective photoelectron circular dichroism (MS-PECD). ${ }^{[10]}$ Since the observed magnitude of the photoelectron circular dichroism scales with enantiomeric excess (e.e.) this approach, in principle, allows for rapid identification and e.e. measurement; indeed subsequent studies using either 
synchrotron ${ }^{[11]}$ or fs laser ${ }^{[12,13]}$ ionization have demonstrated that e.e. determination can be achieved to better than $\pm 1 \%$ in a few minutes.

Limonene (see Figure 1) can adopt a number of conformations, which principally can be separated into those having the isopropenyl tail group in either axial or equatorial positions relative to the ring. Within each of these two groups three rotamers of the tail group are possible, which we can distinguish by the dihedral angle $\angle \mathrm{C}_{a}-\mathrm{C}_{b}-\mathrm{C}_{c}-\mathrm{C}_{d}$. These have been studied with the aid of theoretical calculations ${ }^{[14]}$ which indicate that the axial conformers are disfavoured by at least $6 \mathrm{~kJ} \mathrm{~mol}^{-1}$. At room temperature and below, these are therefore very much minority components of an equilibrium mix, and henceforth we shall discuss just the three equatorial conformers, conveniently labelled Eq-1, Eq-2, and Eq-3. The outer, highest occupied molecular orbital (HOMO) in limonene is predicted to be the ring $\mathrm{C}=\mathrm{C} 2 \pi$ orbital, with the second highest (HOMO-1) being the $\mathrm{C}=\mathrm{C} 1 \pi$ orbital situated in the tail. Energetically, these are quite close and give overlapping contributions to the first $X$ band in the photoelectron spectrum. ${ }^{[15-17]}$ The remaining orbitals are more $\sigma$-bonding in character, and may be expected to lie deeper, but the photoelectron spectroscopy has not, to our knowledge been extended beyond the composite $X$-band. The vacuum ultraviolet (VUV) dissociative ionization has, however, been reported running to somewhat higher ionization energies. ${ }^{[16,18]}$

The VUV absorption spectrum of limonene ${ }^{[15,17]}$ commences at $\lambda \approx 220 \mathrm{~nm}$ with a first Rydberg transition assigned as $2 \pi \rightarrow 3 s$. Brint et al ${ }^{[15]}$ have measured the gas phase absorption circular dichroism $(C D)$ of limonene in this region and Logé and Boes ${ }^{[19]}$ have correspondingly measured the $C D$ (in the ion yield) generated by 2 photon laser ionization of $d$-limonene enantiomer at $213 \mathrm{~nm}$. Two photon resonant $(2+1)$ laser multiphoton ionization, for $\lambda \approx 400 \mathrm{~nm}$, has also been used to investigate the PECD of limonene enantiomers by examining the forward-backward asymmetry in the photoelectron angular distribution that results. ${ }^{[20-22]}$ Although nominally exciting through the same intermediate states at the two photon level, the PECD asymmetry is several orders of magnitude greater than the absorption $C D$ owing to the former effect arising in the pure electric dipole approximation..$^{[23]}$ Such an enhanced asymmetry for VUV PECD, in the $1 \%-40 \%$ range, is by now well established. ${ }^{[24]}$ Nevertheless, these similar MP-PECD results may here be thought a little surprising since the Rydberg character of the intermediate state that is ionized ought to render the ejected photoelectron rather less sensitive to the chiral details of the inner ion core. The interpretation of the multiphoton (MP-)PECD remains challenging. Measurements made with $150 \mathrm{fs}$ duration laser pulses were performed at a small number of wavelengths that coincided with resonant excitation of different vibrational levels of the $2 \pi \rightarrow 3 s$ Rydberg intermediate, and with the $1 \pi \rightarrow 3 s$ intermediate. ${ }^{[20]}$ These showed that a reversal of the sign of the MP-PECD was possible in the latter case. Shorter ( $25 \mathrm{fs}$ ) pulse measurements, but with different proposed assignment of the intermediate levels, recorded similar sign reversals but led these authors to conclude that "PECD is not sensitive to either the electronic character or the strength of the intermediate resonance involved...". ${ }^{[22]}$

In the present paper we provide a detailed PECD study of jet-cooled limonene using single (VUV) photon ionization. In so doing we have the intention to help generate a better understanding of the electron-nuclei dynamics that shape the PECD response of this important molecule. These studies, conducted with synchrotron radiation, should thus assist understanding of the laser MP-PECD. This is desirable to advance the analytical potential of MS-PECD ${ }^{[10]}$ and the novel possibilities for using MPPECD for time-resolved studies of chiral systems. ${ }^{[25]}$ 


\section{Experimental}

\section{CIPO Beamline, Elettra}

Measurements at photon energies spanning the range $16.6 \mathrm{eV}-23.5 \mathrm{eV}$ were performed at the "Circular Polarization" (CIPO) 4.2 beamline at ELETTRA (Trieste, Italy) ${ }^{[26]}$ with the storage ring operating at $2 \mathrm{GeV}$ in top-up mode. Elliptically polarized radiation is produced by an electromagnetic elliptical wiggler, and passes, via a normal incidence monochromator, with a $200 \mu \mathrm{m}$ slit setting, into the experimental chamber. An effusive vapour of commercially supplied enantiopure (>98.5\%) Rlimonene (Sigma-Aldrich) was admitted, following several freeze-pump-thaw cycles of the sample liquid, via a needle. During this the indicated base pressure of the chamber $\left(1 \times 10^{-7} \mathrm{mbar}\right)$ was allowed to rise to $7 \times 10^{-6} \mathrm{mbar}$. Photoelectrons produced by photoionization were detected by a hemispherical analyser (VG - radius $15 \mathrm{~cm}$ ) set at $54.7^{\circ}$ to the synchrotron beam direction, in the vertical plane. Most measurements were made with a pass energy of $3 \mathrm{eV}$ and an energy step increment of $0.01 \mathrm{eV}$, and under these conditions the estimated resolution is 65 meV FWHM. Energy scale calibration was performed by referring peak positions to higher resolution threshold photoelectron spectra recorded at Soleil (vide infra).

The photoelectron circular dichroism (PECD) measurements were taken with alternate photon helicity, achieved by reversing the current in the wiggler's horizontal coils at $0.05 \mathrm{~Hz}$ during each kinetic energy dwell point in the photoelectron energy scans, in order to compensate for any drifts in the gas pressure etc. During this switching the photon beam intensity was monitored with a photodiode and used to normalise the two signal count rates. For the energies employed here the CIPO wiggler does not generate pure circular polarization. It is therefore necessary to correct the observed asymmetries in the electron scans to account for the ellipticity of the radiation.

For pure circular polarization states $p= \pm 1$ (respectively left and right circular polarizations) the expected photoelectron angular distribution takes the form

$I^{\{p\}}\left(\theta_{D}\right)=1+b_{1}^{\{p\}} P_{1}\left(\cos \theta_{D}\right)+b_{2}^{\{p\}} P_{2}\left(\cos \theta_{D}\right)$,

where the $P_{\mathrm{j}}$ are Legendre polynomials, and $\theta_{D}$ is the detection angle with respect to the light beam propagation direction. The parameter $b_{2}^{\{ \pm 1\}}$ is equivalent to $-\beta / 2$ where $\beta$ is the conventional anisotropy parameter measured with linear polarizations. The chiral parameters $b_{1}^{\{+1\}}=-b_{1}^{\{-1\}}$ are the target of a PECD measurement and indicate the magnitude of the forward-backward asymmetry in the photoelectron emission that may be observed with a chiral molecule. ${ }^{[27]}$ Following expressions given by Huang ${ }^{[28]}$ we can generalise Eq. 1 for an arbitrary polarization state and thence obtain:

$$
I\left(\theta_{D}\right)=1+\left|S_{3}\right| b_{1}^{\{p\}} P_{1}\left(\cos \theta_{D}\right)+b_{2}^{\{ \pm 1\}}\left(P_{2}\left(\cos \theta_{D}\right)-3 / 2\left(S_{1} \cos 2 \phi_{D}+S_{2} \sin 2 \phi_{D}\right) \sin ^{2} \theta_{D}\right),
$$

where the $S_{i}$ are Stokes parameters describing the polarization state of the radiation. For our specific detector geometry $\left(\theta_{D}=54.7^{\circ} ; \phi_{D}=0^{\circ}\right)$ Eq.2 simplifies to

$I\left(54.7^{\circ}\right)=1+\left|S_{3}\right| b_{1}^{\{ \pm 1\}} \cos 54.7^{\circ}+\frac{\beta}{2} S_{1}$

Eq. 3 
Hence defining the observed PECD asymmetry, $\Gamma_{\theta_{D}}$, as the normalised dichroism between left and right handed polarizations we find

$\Gamma_{54.7}=\frac{\left(I^{\{+1\}}-I^{\{-1\}}\right)}{\frac{1}{2}\left(I^{\{+1\}}+I^{\{-1\}}\right)}=\frac{2\left|S_{3}\right| b_{1}^{\{+1\}} \cos 54.7^{\circ}}{1+\beta / 2 S_{1}}$.

From the theoretical performance of the CIPO wiggler with the $2.0 \mathrm{GeV}$ storage ring energy we can take predicted values of $\left|S_{3}\right|$ that range from 0.67 at $h v=16.6 \mathrm{eV}$ to 0.85 at $h v=23.5 \mathrm{eV}$. Further assuming $S_{2}=0, S_{1}=\sqrt{1-\left(S_{3}\right)^{2}}$ (i.e. the major axis of the polarization ellipse lies in the orbit plane of the synchrotron and that the unpolarized component of light $\left(S_{4}\right)$ can be neglected) values of $b_{1}^{\{+1\}}$, compensated for the elliptical polarization can be extracted from the measured dichroism by rearranging Eq. 4 . This does, however, require assuming a value for $\beta$, the photoelectron anisotropy parameter. In the absence of measured $\beta$ values we may typically assume that $\beta$ will rise from $\approx 0$ at threshold to 0.5 some few $\mathrm{eV}$ above threshold (as corroborated by theoretical calculations described below).

\section{DESIRS Beamline, Soleil}

Measurements made at photon energies ranging from threshold up to $h v=18 \mathrm{eV}$ were made at the undulator-based DESIRS vacuum ultraviolet beamline, ${ }^{[29]}$ at the SOLEIL synchrotron (St. Aubin, France). The beamline delivered pure circularly polarized light $\left(\left|S_{3}\right|>0.97\right)$ over the whole energy range, as checked with a polarimeter that could be inserted just upstream of the spectrometer. ${ }^{[30]}$ For the lower photon energies ( $h v<15 \mathrm{eV}$ ) employed here an in-line gas filter, filled with $0.25 \mathrm{mbar}$ of $\mathrm{Ar}$, was used to remove higher undulator harmonics, preventing their transmission to the experiment as second order light off the grating. ${ }^{[31]} A$ low-dispersion (200 grooves $/ \mathrm{mm}$ ), high-flux grating $\left(\approx 10^{12}-10^{13}\right.$ photons per second in $0.1 \%$ bandwidth) was selected, providing resolving powers of up to 4000 . In practice however, PECD measurements were made with the monochromator slit settings optimised to control photon flux (avoiding detector saturation) rather than resolution.

Photoionization experiments were conducted using the DELICIOUS $3 \mathrm{i}^{2} \mathrm{PEPICO}$ spectrometer that couples a modified velocity map imaging (VMI) electron analyser with an ion imaging/modified Wiley McLaren time-of flight analyser for ion detection. ${ }^{[32]}$ The VMI analyser provided an energy resolving power of $\sim 4-5 \%$. Recording data in an ion-electron coincidence mode allows the electron energy and angular distributions to be filtered by corresponding ion mass, improving background rejection and consequent $\mathrm{S} / \mathrm{N}$ that could be achieved.

Commercially sourced samples of either R- or S- limonene (Sigma Aldrich) were placed in a room temperature reservoir immediately behind a $70 \mu \mathrm{m}$ pinhole nozzle. The vapour was seeded in 0.5 bar He backing gas and co-expanded through the nozzle, producing a cold seeded molecular beam, which was then double skimmed before entering the main DELICIOUS 3 spectrometer chamber. ${ }^{[33]} \mathrm{In}$ operation with the beam running the main chamber pressure remained $<3 \times 10^{-8} \mathrm{mbar}$.

Our methodology for the recording of PECD using VMI imaging has been fully described previously. ${ }^{[34]}$ In brief, the VMI electron extraction fields were set high enough to collect all the electrons onto the detector. For each selected photon energy and enantiomer, several massselected photoelectron images were acquired by alternating left and right circularly polarized light ( $L C P$ and RCP) and were then combined to obtain a total image ( $L C P+R C P)$ and a difference image 
(LCP-RCP), from which the photoelectron spectrum and PECD $\left(b_{1}\right)$ can be respectively extracted after Abel inversion. ${ }^{[35]}$ Experimental error bars are calculated by applying standard error propagation formulae assuming an initial Poisson distribution on the pixel intensities of the VMI images. Because of the high degree of beamline polarization, as indicated by the Stokes parameters, $S_{3}$, measured at the DELICIOUS spectrometer, in this series of measurements no correction was deemed necessary for the $b_{1}^{\{+1\}}$ values obtained. However, while the R-enantiomer samples were stated as being $>99 \%$ purity with $>98.5 \%$ enantiomeric excess (e.e.) the S-enantiomer samples carried no stated e.e. We therefore commissioned a two-column gas chromatography $\left(\mathrm{GC}^{2}\right)$ chiral analysis of our S- sample that determined an e.e of $78.65 \%$ (3 $\sigma$ s.d. 0.1 ). ${ }^{[36]}$ The observed PECD chiral asymmetry will scale with e.e. ${ }^{[10-12]}$ and we have accordingly adjusted our S-enantiomer PECD measurements to correct for this reduced sample enantiomeric purity.

Threshold photoelectron spectra of the first photoelectron band region were also obtained. For these photon energy-scanned spectra the monochromator exit slits were set to provide a photon resolution of 3-5 meV, and a $3 \mathrm{meV}$ step scan was used, recording additionally the coincident ions. A photon monitor, (photodiode AXUV, IRD Inc.) located right after the spectrometer interaction region permits a photon flux correction to be applied to the recorded count rates. In the basic VMI threshold electron photoelectron technique ${ }^{[37,38]}$ (TPES) a small extraction field focuses electrons with a kinetic energy approaching zero into the center of the detector, while fast electrons are strongly discriminated and their remaining contribution, evidenced by the off-center detector signal, can be corrected for. In our modified slow photoelectron (SPES) technique ${ }^{[39]}$ photoionization matrices mapping the electron intensity as a function of both photon and electron kinetic energy are generated from the scan. This treatment permits the inclusion of faster electrons, with appropriate adjustment of their apparent ionization energy, into the SPES result, with the benefit a correspondingly improved $\mathrm{S} / \mathrm{N}$ but minimal degradation of the threshold energy resolution. For the results shown here electrons with kinetic energy $\leq 30 \mathrm{meV}$ were accepted, and the overall estimated resolution is $\sim 20 \mathrm{meV}$. Overall calibration of the absolute photon energy was accomplished by monitoring atomic $\mathrm{Kr}$ absorption lines arising from the use of $\mathrm{Kr}$ in the upstream harmonic gas filter.

\section{Computational}

In this paper we have concentrated on the lower energy equatorial conformers of limonene, as the higher energy axial forms ${ }^{[40]}\left(\sim 7 \mathrm{~kJ} \mathrm{~mol}^{-1}\right)$ are unlikely to be significantly populated at room temperature or below. Geometry optimisations, including harmonic vibrational frequency analysis, for the equatorial forms Eq-1 - Eq-3, distinguished by the dihedral angle $\angle a-b-c-d$ (see Fig. 1 ) of the isopropenyl tail group were performed for both the neutral molecules and the corresponding ground state cations using the Gaussian-09 software. ${ }^{[41]}$ All structures were confirmed as true minima by checking the absence of imaginary frequencies. Relative energies of conformers and of ion/neutral pairs can then be assessed at a given level of theory by comparing absolute electronic energies with zero point vibrational energy corrections applied. A variety of methods (CASSCF, MP2, DFT) and basis sets were compared. Unrestricted SCF was used for the open-shell cation calculations, but no significant spin contamination issues were detected. The CASSCF calculation used a small active space comprising the $\mathrm{C}=\mathrm{C} \pi$ orbitals.

Vertical ionization energies for the conformers Eq-1-Eq-3 were estimated by outer valence Green's function (OVGF) calculations. ${ }^{[42,43]}$ Franck-Condon (FC) simulations of vibrational structure in the 
HOMO PES band were performed using FC-Lab II software. ${ }^{[44]}$ For this we use scaled harmonic vibrational parameters obtained for the neutral and cation state at a given level of calculation (method/basis). Calculations of the FC factors include Duschinsky rotation and allow for excitations of all modes from the vibrationless ground state (except where otherwise noted), including combination bands of up to three normal modes.

Selected key results are given, and compared with literature results, in Tables 1,2, and 3, but a full summary of our calculations is provided in Supporting Information. It was noted that the density functional methods in general, and the B3LYP functional in particular, gave much smaller dihedral angles for the optimum Eq-2 cation geometry compared to other methods, and to the neutral structure. The M11 functional proved the exception to this. The methods all produced quite variable dihedral angles for both the neutral and cation Eq-3 structure. This sensitivity appears to reflect a rather flat, broad minimum at the Eq-3 equilibrium geometry, as partially seen in the $\angle a-b-c-d$ torsional potentials ${ }^{[45]}$ (see also Fig. S1, Supporting Information), and also evidenced in the much lower (harmonic) torsional vibration frequencies, $v_{1}$, calculated for Eq-3 conformers.

Photoelectron angular distribution parameters (the anisotropy parameter $\beta$ and chiral PECD parameter $b_{1}^{\{+1\}}$ ) were calculated using the CMS_X $\alpha$ method following procedures we have previously described. ${ }^{[46-50]}$ These computations were performed at the fixed MP2/cc-pVDZ optimised R-enantiomer geometries of the three equatorial conformers. Multiple scattering equations for the continuum electron are solved in a model potential consisting of overlapping atomic spheres (set at 0.85 of the so-called Norman atomic radius ${ }^{[51]}$ ) with a spherical harmonic angular basis truncated at $I_{\max }=18,5,3$ (referring respectively to the spherical region outside the molecule, the $C$ atomic regions, and the $\mathrm{H}$ atomic regions). For the initial state a smaller basis $\left(I_{\max }=6,3,1\right)$ sufficed.

\section{Results}

\section{Photoionization}

Figure 2 shows an overview of outer valence region photoelectron spectrum (PES) of limonene, recorded at Elettra with $h v=19.5 \mathrm{eV}$. Also marked are the predicted positions of vertical ionization energies, obtained from OVGF/6-311G** calculations. As known from previous work ${ }^{[16,17]}$ the first PES band spans the expected HOMO and HOMO-1 ionizations. There is then an interband gap at $\sim 9.8 \mathrm{eV}$ before a structured further band extending to above $16 \mathrm{eV}$. Considering just the Eq-1 conformer vertical ionization energies, there seems a clear correlation between maxima at $10.4 \mathrm{eV}$ and $11 \mathrm{eV}$ suggesting a simple assignment, although this becomes less clear when the HOMO-3 ionization of conformers Eq2 and Eq3 is also viewed. Above $11.4 \mathrm{eV}$ the spectrum is clearly too congested to readily identify individual orbital contributions, although the broader maxima centred at $12.5 \mathrm{eV}$ and in the range $13.5-15 \mathrm{eV}$ do nevertheless appear to correlate with predicted high orbital densities, before falling to a final shoulder at $15.8 \mathrm{eV}$ that corresponds with a better separated single orbital prediction.

An example of the ion ToF spectra recorded with DELICIOUS 3 using a supersonic beam of limonene is shown in Figure 3. The parent ion region is shown as an expanded inset where, as well as the monomer (136.2 amu) a second (137.2 amu) and third (138.2 amu) peak can be attributed to limonene molecules possessing one or two ${ }^{13} \mathrm{C}$ at natural abundances ( $11 \%$ and $1 \%$ respectively). At photon energies $h v=9.5 \mathrm{eV}$ and below, the ToF spectra are essentially the same, showing no fragmentation, with perfectly flat baselines extending up to the parent ion region. Only at $9.8 \mathrm{eV}$ and then $10.0 \mathrm{eV}$ do we start to discern evidence of ion fragmentation. As seen in Fig. 3, at 10.0 eV there are very weak $(<<1 \%)$ peaks at $93 / 95$ amu and 121 amu. The complete lack of fragmentation for $h v$ 
$\leq 9.5 \mathrm{eV}$ contrasts with our own earlier photoionization studies of limonene $^{[16]}$ and the more recent molecular beam work of Fang et al. ${ }^{[18]}$ which both show more significant amounts of "94" amu, 121 $\mathrm{amu}$, and other fragment masses, all with apparent onsets in the $9 \mathrm{eV}-9.3 \mathrm{eV}$ region. However, in both these earlier works the molecular beam expansion was in argon, and used pre-heating of the limonene sample reservoirs (to respectively $110^{\circ} \mathrm{C}$ and $50^{\circ} \mathrm{C}$ ) to generate increased molecular vapour pressure. We infer that much more efficient cooling of vibrational modes may be being achieved in the current experimental arrangement (double skimmed expansion in $\mathrm{He}$, room temperature reservoir). Indeed, at the reservoir temperatures used in earlier works there may be $\sim 10 \%$ of the more energetic axial conformers ${ }^{[14]}$ and it is feasible that this population may not be efficiently relaxed to the equatorial forms in the molecular beam expansions, leading in turn to conformationally excited cation production.

Using B3LYP/6-311++G calculations Fang et al. ${ }^{[18]}$ are able to suggest relatively low thermodynamic thresholds for $\mathrm{m} / \mathrm{z} 94$ and $\mathrm{m} / \mathrm{z} 121$ dissociation channels, but with transition states that create significant kinetic barriers. The asymmetrically skewed fragment ToF peak shapes seen for $h v=10.0$ $\mathrm{eV}$ in Fig. 3 do indeed suggest these minor $\mathrm{m} / \mathrm{z} 94$ and $\mathrm{m} / \mathrm{z} 121$ fragments may result from slow, metastable dissociation, which would confirm some kinetic barrier.

While we have no quantitative estimate of the molecular beam temperature achieved in the current work we can deduce from the evident suppression of thermal fragmentation in the cation that there is more efficient vibrational cooling than hitherto achieved. Moreover, because the first two monomer cation states resulting from the outermost $\mathrm{C}=\mathrm{C} \pi$ orbital ionizations are unfragmented and fully stable for $h v \leq 9.5 \mathrm{eV}$, the use of coincidence detection to select only electron signals correlating with $\mathrm{m} / \mathrm{z} 136$ parent ion will not discriminate against HOMO and HOMO-1 ionizations, but will be advantageous in reducing any VMI image background associated with slower electron production from deeper lying orbitals or other unrelated processes (background gas, scattered light-induced photoemission, etc...). Such filtering is not possible for the non-coincident data recorded for higher photon energies at Elettra.

The calibrated $X$-band photoelectron spectrum (SPES) of jet cooled limonene is shown in Figure 4 where the previously published ${ }^{[17]} \mathrm{He}$ I photoelectron spectrum of a room temperature sample is included for comparison. The latter recording has a stated resolution of $25 \mathrm{meV} \mathrm{FWHM}$, and displays exceptional S/N. As judged by the width of the principal peaks, the current SPES has identical resolution. Earlier studies of this PES band ${ }^{[15-17]}$ have established that ionization of both the HOMO (ring $\pi$ orbital) and HOMO- 1 (isopropenyl tail $\pi$ orbital) are overlapping, with the onset of the latter not clearly distinguished. The positions of the calculated OVGF/6-311G $(d, p)$ vertical ionization energies (Table S5, Supporting Information) are, however, indicated on this figure.

Experimentally, there is excellent agreement between the positions of the principal progression of peaks in the SPES and He I PES spectra (8.524 eV, $8.695 \mathrm{eV}, 8.881 \mathrm{eV}$ in the SPES vs. $8.521 \mathrm{eV}, 8.695$ $\mathrm{eV}$, and $8.867 \mathrm{eV}$ in the He I PES $\left.{ }^{[17]}\right)$. There are, however, discrepancies associated with the secondary progression of smaller peaks seen in the He I PES (labelled $a, b, c, d$ ). As seen in Fig. 4 these may be either much attenuated (peak $b$ ), or even absent ( $c$ and $d$ ) in the SPES. These differences might be attributable to resonant autoionization contributing to the threshold spectra, contrasting with direct ionization processes in the He I spectrum, but is far more likely associated in some way with the very different sample inlet conditions (jet cold SPES vs. room temperature He I PES). In an effort to test this postulate we attempted to reproduce the He I PES ${ }^{[17]}$ while using an effusive source at Elettra (see Fig. 4). Unfortunately, inspection reveals a lower resolution was achieved in this case, and while the small peaks were again missing this is deemed of limited significance; a small amount of data manipulation shows that this is an expected consequence as the valleys between major 
peaks fill in at lower resolution. Nevertheless, given the evidently similar resolution between the SPES and He I spectra, we cannot yet discount temperature as a likely source of their differences.

A Fourier transform microwave (FTMW) rotational spectroscopy experiment ${ }^{[45]}$ has previously found that upon moderate cooling in supersonic molecular beam only the Eq-1 and Eq-2 conformers were detectable; the Eq-3 conformer was absent. This accords with calculated torsional potentials (Ref. [45], and Supporting Information) which predict only a low barrier between Eq-2 and Eq-3. It was therefore concluded in the FTMW experiments that any Eq-3 population was efficiently relaxed into the Eq-2 conformer. ${ }^{[45]}$ Considering the different molecular beam conditions employed for this FTMW study (expansion in $\mathrm{Ne}, 70^{\circ} \mathrm{C}$ reservoir) and our present work (double skimmed expansion in $\mathrm{He}, 20^{\circ} \mathrm{C}$ reservoir), and bearing in mind the preceding discussion of the ion ToF results, it seems highly probable that our molecular beam is at least as cold, and we henceforth assume that there is no Eq-3 conformer present in our photoionization experiments at Soleil. This may thus be an explanation for the reduction of peaks $b, c, d$ observed between the He I PES and SPES in Fig. 4.

To pursue this we have calculated Franck-Condon (FC) simulations of the vibrational structure expected in the HOMO $2 \pi^{-1}$ ionization. Fig. 4 includes the simulated $0 \mathrm{~K}$ spectrum for a single conformer, Eq-1, using MP2/cc-pVDZ calculated vibrations, and simulations for all three equatorial conformers are compared (plotted with slightly higher resolution to better reveal structure) in Figure 5A. Generally, simulations obtained using vibrational parameters obtained from various levels of calculation (listed in Supporting Information) provide very similar results, the exceptions being those density functional calculations with the B3LYP functional which lack such close resemblance to the jet cooled SPES for Eq-1 (and suggest vanishingly small FC factors for Eq-2).

The FC simulations compared in Fig $5 \mathrm{~A}$ are sufficiently similar to suggest that changes in the Eq-1:Eq2 ratio would be imperceptible in the spectra, but nor is it immediately obvious how a reduction in the Eq-3 population, as proposed above, might produce the observed changes in the experimental spectra. We therefore offer one tentative rationalization that assumes conformer dependent adiabatic ionization energies. In particular, we postulate that $\mathrm{l}_{\text {adib }}$ for $\mathrm{Eq}-3$ may be some $0.08 \mathrm{eV}$ higher than the others. Efforts to estimate absolute values of ladib $_{\text {dib }}$ by considering $\Delta E_{\text {E0+ZPE }}$ produce too much variability (see Supporting Information) to permit confident predictions, but nevertheless an $80 \mathrm{meV}$ offset is plausible. A consequence of this would be that the peaks of the prominent progression in Eq-3 would be displaced and fall between those of Eq-1/Eq-2. A simple visualisation of this is shown in Fig. 5B which combines the FC conformer simulations as just discussed, assuming a relative conformer weighting compatible with the expected $300 \mathrm{~K}$ Boltzmann populations (Table 2). It can thus be seen that, given this additional assumption about $l_{\text {adib }}$ offsets, the differences between the Hel PES and SPES are consistent with removal of the Eq-3 conformer in the jet cooled Soleil experiments.

The single conformer Eq-1 simulation (i.e. explicitly excluding any Eq-3 contribution) shown in Fig. 4 in fact provides rather satisfying agreement with the jet cooled SPES (although the following comments will apply equally to the Eq-2 conformer simulation which is virtually identical, as can be seen in Fig. 5A). Not unexpectedly, the experiment and simulation diverge above $8.85 \mathrm{eV}$ where contributions from the second $1 \pi^{-1}$ ionization can also be expected.

From the simulated stick spectrum the principal vibrational progression evident in the experimental PES can be assigned to one involving mode 55 (essentially stretching of the ring $\mathrm{C}=\mathrm{C}$ double bond, scaled frequency $=1447 \mathrm{~cm}^{-1}$ ) appearing in combinations with $0-3$ quanta of the low frequency deformation mode $v_{2}$, and methyl torsion, $v_{3}$. Lying to higher energy than these clusters of transitions is a second short and weaker progression in $v_{55}$ built on a combination of single quanta in 
$v_{3}$ and $v_{17}\left(v_{3}\right.$ and $v_{18}$ in Eq-2). In the realistically broadened vibrational envelope plotted in Fig. 4 only the first member of this progression built on skeletal vibrational modes (scaled frequencies 634 $-664 \mathrm{~cm}^{-1}$ ) can be readily discerned as a minor peak nestled between the first major peaks.

However, this exactly matches and explains the residual peak $b$ seen in the SPES, and the absence of peaks $c, d$.

In preparing Fig. 4 the harmonic scaling factor (0.91) and ionization energy scale origin (8.505 eV) applied to the FC simulation were determined to best match the experimental spectrum. The origin 0-0 transition is not predicted as the most intense transition in the low energy region, meaning that the observable peak maximum seen in the simulation is shifted to slightly higher energy. We therefore conclude that true adiabatic ionization energy, $\mathrm{l}_{\text {adib }}=8.505 \pm .005 \mathrm{eV}$.

The vibrational analysis by Smialek et al ${ }^{[17]}$ reached the same identification of the vibrational modes excited as here, but otherwise differs in detail. In particular, their vibrational simulations suggested FC factors for Eq-1 and Eq-3 that are 3-4 orders of magnitude less than for Eq-2, so that any contribution from ionization of these conformers could be discounted. This seems to be a consequence of the much larger changes in the Eq-1 and Eq-3 dihedral angles upon ionization, $20^{\circ}$, predicted by their MP2-ZAPT (restricted HF reference functions) geometry optimization and is not replicated in the methods we have followed (see Table 1 and Supporting Information).

Despite the good agreement between the current FC model and the jet cooled SPES in Fig.4, it is evident that there is some electron intensity, observable in both the Hel PES (peak $a$ ) and the SPES despite their temperature differences, that lies below the adiabatic $0-0$ origin, $8.505 \mathrm{eV}$. This has still to be explained. The FC simulations published by Smialek et al. ${ }^{[17]}$ included hot band transitions, but they concluded that at no feasible temperature could peak $a$ be fitted as an excitation from a vibrationally excited neutral. Similarly, when attempting hot band simulations we also found that peak $a$ was too intense to be fitted with any feasible temperature. In fact, comparing the SPES and Eq-1 OK simulations, it is clear that the observed peak $a$ is sitting on a more diffuse feature below the 0-0 adiabatic ionization which possesses a quite significant integrated photoelectron count.

Smialek et al. ${ }^{[17]}$ then postulated that $a$ may result from dimer formation. The current jet-cooled conditions in the SPES recording might be thought even more amenable to dimer formation. Unfortunately, the experimental ion extraction fields we employed did not allow for direct detection of cluster masses so that we cannot fully test this hypothesis. We can, however, propose an alternative, but equally speculative, explanation. That is to suppose that the feature at $a$ is in fact associated with the $1 \pi^{-1}$ ionization. While it seems fairly well established that the vertical ionization energies lie $2 \pi^{-1}<1 \pi^{-1}$, as already noted there is no reliable estimation of adiabatic ionization energies of which we are aware. In the event that the vertical ionization region is significantly displaced from the $1 \pi^{-1}$ cation equilibrium geometry the $1 \pi^{-1}$ electron spectrum could have a long weak tail leading down to the true adiabatic ionization energy.

\section{Photoelectron circular dichroism}

Overviews of PECD recorded by VMI imaging at various photon energies with jet-cooled limonene in the DELICIOUS 3 apparatus are given in Figures 6 and 7. Fig. 6 shows the $10 \mathrm{eV}-12 \mathrm{eV}$ ionization energy range $(A / B$ band region identified in Fig. 2). At ionization energies above $10.5 \mathrm{eV}$ the PECD recorded with low photon energies $(\leq 12 \mathrm{eV}$ ) clearly strongly diverges from the higher photon energy measurements, but then these do not show significant differences from one another. Below $10.5 \mathrm{eV}$ ionization energy, from the low energy side to the centre of the $A$-band, one sees a much more systematic spreading of the PECD with increasing photon energy. It is not simple to correlate this behaviour with individual orbital structure. From the OVGF calculations the $A$-band peak in the 
PES can be fairly clearly assigned as the $3^{\text {rd }}$ orbital ionization, but the PECD varies markedly across this band. The $B$-band peak in the PES looks quite distinct, but as already noted the different conformers cloud the assignments to the experimental peaks at ionization energies above $10.5 \mathrm{eV}$. While the jet-cooling employed for the PECD measurements may allow Eq-3 conformer contributions to be discounted ${ }^{[45]}$ it remains the case that there is no strong correlation of observed PECD with the indicated orbital ionization energies other than perhaps the above noted divergence of the lowest photon energy measurements for ionization energies above $10.5 \mathrm{eV}$.

Fig. 7 similarly shows VMI-PECD measurements made at lower photon energies covering the $X$-band region, and one of the VMI-PES measurements is over-plotted to help indicate where the vibrational structures, discussed above, lie. While the higher photon energies allow the upper extent of the viewed ionization energy to be extended further across the PES band, the measurements are remarkably similar and show a strong correlation of the PECD peaks and troughs, swinging between positive and negative values, with vibrational PES peaks and valleys, without any very pronounced photon energy dependence.

This behaviour is examined more thoroughly using Figure 8 that shows the $h v=9.5 \mathrm{eV}$ VMIPES/PECD measurement made with R-limonene, and additionally includes a FC PES simulation to show the anticipated HOMO $2 \pi^{-1}$ vibrational peaks. (Here we use the M11/cc-pVDZ simulation to show it is effectively interchangeable with the MP2 simulation in Fig. 4.) There is clearly a strong modulation of the observed PECD at the position of each of the calculated vibrational peaks.

Especially interesting is the behaviour at the third peak $(8.85 \mathrm{eV})$. Here the PECD starts to rise at the PES peak position, then suddenly drops before seemingly resuming modulation at the position of the $4^{\text {th }}$ predicted peak ( $\sim 9.05 \mathrm{eV}$ ). From Table 3 it can be seen that the predicted HOMO- $11 \pi^{-1}$ vertical ionization energy is $\sim 8.9 \mathrm{eV}$, which leads us to conclude that the sharp drop is attributable to the new contribution coming from this second ionization. Hence the underlying trend of the coarse ( $R$ enantiomer) PECD, averaged through the vibrational maxima, would appear to switch from positive to negative values on moving between regions were HOMO and then HOMO-1 ionizations dominate. Although there is no fundamental necessity for this, we note that such flips in PECD asymmetry have often been observed between adjacent HOMO and HOMO-1 ionization peaks, showing how PECD is orbital-dependant (initial state effect). ${ }^{[11,49,52,53]}$ Above $9 \mathrm{eV}$ ionization energy, where the recorded PECD represents some superposition of the first and second orbital ionizations, there is evidence for continued vibrational modulation of the former contribution.

We have recently examined and analysed vibronic effects in the PECD of small oxirane molecules ${ }^{[2,3]}$ in near-to-threshold ionization. Such vibrational sensitivity in the chiral terms of the photoelectron angular distribution is attributed to an enhanced phase dependence in the chiral photoionization dynamics caused by vibrational motions. ${ }^{[3,4]}$ The current limonene $X$-band observations reveal an even stronger modulation and so provide a unique opportunity, to reveal such electron-nuclear motion coupling in a considerably larger molecule.

It could be suggested that any prominence of vibronic PECD effects in near threshold ionization owes something to the increased sensitivity of low energy photoelectrons to scattering by the chiral molecular potential. Indeed, the original finding of vibrationally dependent PECD in methyl oxirane ${ }^{[1]}$ was made at much higher photon energy but was a relatively weaker modulation than in the near threshold results. Pragmatically, a reason for focussing on the near threshold region with VMI detection is that under these conditions electron energy resolution as low as $10 \mathrm{meV}$ is possible, allowing best resolution of vibrational structure. Unfortunately, the resolving power, $E / \Delta E$, is constant in a VMI instrument. Therefore, as one moves to higher photon energies, in turn necessitating an increase of repeller potential to maintain capture of faster photoelectrons, the 
absolute resolution is diminished ( $\triangle E$ increases) and vibrational resolution is lost. This is evident in Figure 9 showing the $X$-band VMI-PES and PECD recorded at $h v=18 \mathrm{eV}$. The VMI-PES is now just a smooth hump and the VMI-PECD, although maintaining a decrease from left to right across the band, is featureless.

To overcome these limitations of the VMI technique, measurements were continued to higher photon energies using the constant resolution dispersive electron analyser on the CIPO beamline. These results for $h v=18 \mathrm{eV}$ are directly compared in Fig. 9. In general, the PECD magnitude is in excellent overall agreement with the $\mathrm{VMI}$ measurement (allowing that the opposite enantiomer was used), lending confidence in the reproducibility of the measurements between the techniques. The principal 4-peak structure of the $X$-band PES is now maintained, but there are only weak indications of vibrationally correlated structuring in the PECD curve, with only the dip at $9.1 \mathrm{eV}$ comfortably exceeding the error bars established in the analysis. With such small differences due to the improved resolution it is impossible to identify any further discrepancies which might be due to the different inlet temperatures between the CIPO and DESIRS setups.

Figure 10 shows the Elettra measurements continued up to our maximum photon energy, $21.4 \mathrm{eV}$. It is immediately apparent that between $h v=19.5 \mathrm{eV}$ and $h v=20.5 \mathrm{eV}$ the sign of the coarse PECD switches from being overall positive (for the R-enantiomer) to overall negative. Any vibrational modulation from $18 \mathrm{eV}$ (as already noted) to $20.5 \mathrm{eV}$ remains very weak. However, the measurement at $21.4 \mathrm{eV}$ has rather convincing dips at the position of the first three principal vibrational PES peaks, despite relatively large error bars, followed by a distinct shift towards zero above $9 \mathrm{eV}$. In other words, it displays a near inverse of the trends noted in low photon energy measurements (Figs. 6, 7).

In an effort to gain further insight into the relative contribution and behaviour of the HOMO $2 \pi^{-1}$ and HOMO-1 $1 \pi^{-1}$ orbital ionizations the following procedures have been followed. Between $h v=11 \mathrm{eV}$ and $h v=18 \mathrm{eV}$, where the VMI-PES lack well-resolved vibrational structure, it has been found possible to rather successfully fit two gaussian functions to the X-band profile. An example is shown in Fig. 9. We choose to assume that these are then somewhat indicative of the position, width and relative intensity of the real $2 \pi^{-1}$ and $1 \pi^{-1}$ bands. Weighting the PECD curves with these two fitted gaussians we are then able to extract two mean PECD values. This approach can be continued for the CIPO PECD data at higher photon energy, although gaussian fits become less good with improved resolution of PES vibrational structure.

These fit-derived estimates for the $2 \pi^{-1}$ and $1 \pi^{-1}$ mean PECD are plotted as a function of photon energy in Figure 11. Also included for the HOMO ionization are the PECD values directly read off the first two principal vibrational peaks for the photon energies appearing in Fig. 7. These appear to follow the same trend approaching the photoionization threshold as do the Gaussian fit derived values. Attempts to model and reproduce the PECD behaviour for these two orbitals, and thereby gain possible insight into the conformer populations, using CMS-X $\alpha$ calculations at fixed equilibrium geometries unfortunately provide very modest success. A major discrepancy is that the magnitudes of the calculated PECD are significantly greater than experiment. This may not be so surprising at near threshold energies, where theoretical limitations are recognised to likely be more severe, but is also evident in the mid-range, $\sim 15 \mathrm{eV}$, examined here. Of course it cannot be ruled out that the limited resolution and rather indirect data extraction method may introduce a bias towards delivering systematically attenuated experimental values. The conformer dependent calculations are nevertheless reproduced in Fig. 11, but only after uniformly scaling magnitudes down by $50 \%$ to fit the same plots. 
What can be seen unambiguously from this presentation of calculated PECD in Fig. 11 is the relative conformer dependent behaviour. For the HOMO $2 \pi$ orbital the conformer variations are initially very small and even going above $15 \mathrm{eV}$ they remain fairly similar. Such conformer invariance in the predicted PECD is a little unexpected. ${ }^{[24,49,54-57]}$ In contrast the HOMO-1 $1 \pi$ orbital predictions show far greater conformer dependent variability, far more in keeping with prior generalised expectations. The lack of a strong $2 \pi$ dependence might be thought not unreasonable given that the initial orbital is localised at the $\mathrm{C}=\mathrm{C}$ double bond in the ring, and the conformers differ only by rotation of the remote tail grouping. However, in a somewhat analogous cyclic terpenoid system, carvone, a significant variation of PECD from a highly localised ring C1s orbital with rotation of a similarly remote tail grouping was noted. ${ }^{[58]}$

Regardless of the forgoing discussion, the quantitative agreement achieved between theory and experiment is here unusually poor, and only at energies $h v \geq 15 \mathrm{eV}$ could it be argued that the trends, including the switching sign of the non-vibrationally resolved HOMO PECD, are at least qualitatively correct once an appropriate average of the Eq-1 and Eq-2 conformers is considered and the absolute values are halved.

\section{Conclusions}

The limonene X-band spectra provide the most intense and striking examples of PECD modulation across a photoelectron band observed to date, displaying behaviour which is both very regular and clearly correlates with vibronic features in the PES. Earlier observations of such clear vibronic influences have been made with smaller chiral molecules (methyl- ${ }^{[1-3]}$ and trifluoromethyl-oxiranes ${ }^{[3]}$ ) and it may be thought unusual for such well-resolved, intense vibrational structures to be observed in either the photoelectron spectrum or PECD spectrum of much larger molecules. Nevertheless, and quite generally, even unresolved vibrational excitations clearly control the observable PES band profile and have the potential to modify the observed PECD. Our understanding is that the enhanced sensitivity of the PECD effect to small variations in the scattered photoelectron phase, in turn a consequence of the dynamics of the molecular nuclei framework, can make for profound fluctuations in the PECD angular asymmetry linked to vibrational excitations. ${ }^{[3,4]}$ Considered another way, it is not unusual for PECD asymmetry to vary across a vibrationally un-resolved photoelectron band profile for a well-isolated orbital ionization, and we hence suspect such trends may be attributable to unresolved vibronic influences.

The first few major peaks in the limonene $\mathrm{X}$-band PES are readily assigned, by intuition or more quantitative $\mathrm{FC}$ simulations, as principally a progression in the $v_{55}$ ring $\mathrm{C}=\mathrm{C}$ bond stretching mode in the $2 \pi^{-1}$ cation state. It is clear that peaks in the PECD intensity are closely matched to these PES vibrational peaks - not just for the low energy, near threshold ionization, but also for much faster electrons formed with more than $10 \mathrm{eV}$ kinetic energy by the action of more energetic photons. However, the FC simulations also make clear that these $v_{55}$ mode excitations occur in combination transitions with various other lower frequency modes, and that underlying this there is a forest of much weaker transitions (see Fig. 4) involving other members of the 72 vibrational modes possessed by this molecule. More particularly, contributing to the intensity filling in between the major peaks, the simulations identify excitations of skeletal modes $v_{17}, v_{18}$. For the better resolved low photon energy measurements ( $h v<9.5 \mathrm{eV}$ - see Fig. 7), the PECD measured in these skeletal excitation "valleys" dips back not to an achiral baseline of zero but further, switching sign i.e. a reversal of the forward-backward asymmetry relative to the main peak values. Thus, even though far from fully resolving individual vibrational quantum state excitations, we can infer profound differences in the chiral photoionization dynamics when strong (intense) and weakly excited vibrational modes are 
compared. Such a flipping of the PECD asymmetry between vibrational PES peaks with dissimilar FC factors was the most dramatic observation made, and rationalised, in the oxirane studies ${ }^{[2,3]}$ which benefited from a lower vibrational state density.

In the case of the current limonene observations, there are a number of factors other than just the high density of unresolvable, weak vibrational excitations that inhibit a more quantitative analysis of the vibronic PECD influences. These we consider in turn.

The first concerns uncertainty over conformer populations. While we can cite the FTMW experiments of Moreno et al $^{[45]}$ to support our expectation that conformer Eq-3 is effectively frozen out, making no contribution in our VMI/PES/PECD measurements, the exact remaining Eq-1 : Eq-2 conformer ratio in the non-Boltzmann molecular beam environment remains uncertain. FC simulations suggest these conformers have very similar, effectively indistinguishable, PES profiles. Since, however, there is nothing to suggest any offset in the ionization energies of these two conformers their spectra may simply be superimposed, with the conformer ratio, whatever it is, being effectively constant moving through both peaks and valleys in the spectrum. Hence the corresponding modulation of the PECD would not be caused by conformer differences, although the absolute magnitudes may well be expected to change with conformer ratio.

The situation is different for the high photon energy measurements made with an effusive source at Elettra. Here a Boltzmann thermal equilibrium applies, and exact conformer ratios, including for Eq3 , can be reliably estimated. Furthermore, if our hypothesis of a small offset in the Eq-3 ionization energy were to be correct, PECD measurements made away from the principal peaks, in the "valleys" between, would become more indicative of the Eq-3 conformer. The PECD modulation in the effusive measurements (Fig. 10) is less pronounced than in the VMI case. Most likely this could be attributed to differences in the experiments' resolution, but it is conceivable that the Eq-3 conformer contributions also influence the inter-peak gap modulation.

A second complication concerns the second overlapping $1 \pi^{-1}$ ionization to the high energy side of the $X$ band. Again, higher level calculations are seemingly required to reliably establish the adiabatic ionization energy. There does appear, however, a rather strong influence on the underlying PECD (that is considering overall coarse trends across some multiple vibrational peaks) that for the Renantiomer switches from positive to negative values where the dominant ionization channel may be assumed to switch from $2 \pi^{-1}$ to $1 \pi^{-1}$. A somewhat more ambitious treatment attempts to attribute the underlying PECD for these channels based upon an X-band profile analysis and thus to study the HOMO and HOMO-1 PECD as a function of photon energy (Fig. 11). It is also then feasible to perform CMS-X $\alpha$ fixed equilibrium geometry PECD calculations for comparison, but these provide very limited success as theory and experiment differ quantitatively by at least a factor of two. Of course this may be not so much a theoretical failing as a reflection of the rather imprecise method of experimental data analysis, itself something of a consequence of the uncertainties identified in the preceding paragraph. It also rather begs the question whether there is any coupling, rather than a simple superposition, of the close-lying $2 \pi^{-1}$ and $1 \pi^{-1}$ states. Traditional spectroscopic discussions identify the phenomenon or concept of intensity borrowing as a manifestation of full vibronic state coupling, but the implications for photoelectron angular distributions are not so well developed or mature.

A final possible complication stems from the possible presence of clusters in the beam. While an indirect hint of this might be found in the velocity spread of the limonene ions measured from the ion images, direct observation of clusters in the ToF was not possible due to experimental restrictions. 
Despite, or because of, its spectroscopic complexity limonene presents an interesting challenge for further investigation. In a complementary study using $(2+1)$ resonance enhanced ionization of limonene by fs laser pulses ${ }^{[20]}$ we were able to show how, by exploiting the $\Delta v=0$ propensity rule for ionization out of a Rydberg intermediate state, it was possible to exert some control over the final vibrational state in the cation and hence to influence the PECD by pumping through different intermediate state levels. These opportunities were at the time constrained by limited tunability and the relatively large bandwidth of the fs laser pulses, but these can be improved upon using longer pulse durations to provide more complete spectroscopic selectivity. This then brings a further potential for conformer-selective ionization by resolution of the different conformer excitations to the intermediate around their respective origins. This approach was elegantly demonstrated in a study of ion yield $C D$ in gas phase ephedrine enantiomers ${ }^{[59]}$ to reveal very different $C D$ of the individual conformers. Using a similar approach in PECD investigations is fully feasible. Taken together such spectroscopic improvements in vibrational and conformational selectivity could provide a major step in fully unravelling the limonene chiral photoionization dynamics, and hence contribute to the ongoing development of reliable theoretical techniques for modelling and interpretation of the PECD phenomenon.

\section{Acknowledgements}

This research has been supported (HG) by the EU H2020 MSCA ITN "Aspire".

We acknowledge the provision of beamtime and financial support by EC Seventh Framework Programme-Access to Research Infrastructures-CALIPSO (for Soleil beamtime Proposal No. 20140139) and by Elettra (Proposal 20150319) and we thank the technical staff at these facilities for their support and for the smooth operation of each facility.

We should like to thank Iuliia Myrgorodska and Uwe Meierhenrich for the measurement by GC $\times G C$ of the enantiomeric purity of our S-limonene sample.

We are grateful for access to the University of Nottingham High Performance Computing Facility in support of the computational effort. 


\section{References}

[1] G. Contini, N. Zema, S. Turchini, D. Catone, T. Prosperi, V. Carravetta, P. Bolognesi, L. Avaldi, V. Feyer J. Chem. Phys. 2007, 127, 124310.

[2] G. A. Garcia, L. Nahon, S. Daly, I. Powis Nat. Commun. 2013, 4, 2132.

[3] G. A. Garcia, H. Dossmann, L. Nahon, S. Daly, I. Powis ChemPhysChem. 2017, 18, 500-512.

[4] I. Powis J. Chem. Phys. 2014, 140, 111103.

[5] W. Song, M. Staudt, I. Bourgeois, J. Williams Biogeosciences. 2014, 11, 1435-1447.

[6] N. Yassaa, I. Peeken, E. Zollner, K. Bluhm, S. Arnold, D. Spracklen, J. Williams Environ. Chem. 2008, 5, 391-401.

[7] J. Williams, N. Yassaa, S. Bartenbach, J. Lelieveld Atmos. Chem. Phys. 2007, 7, 973-980.

[8] A. Steinemann Build. Environ. 2017, 111, 279-284.

[9] C. M. Wang, B. Barratt, N. Carslaw, A. Doutsi, R. E. Dunmore, M. W. Ward, A. C. Lewis Environ. Sci.-Process Impacts. 2017, 19, 528-537.

[10] M. M. Rafiee Fanood, N. B. Ram, C. S. Lehmann, I. Powis, M. H. M. Janssen Nat. Commun. 2015, 6, 7511.

[11] L. Nahon, L. Nag, G. A. Garcia, I. Myrgorodska, U. Meierhenrich, S. Beaulieu, V. Wanie, V. Blanchet, R. Géneaux, I. Powis Phys. Chem. Chem. Phys. 2016, 18, 12696-12706.

[12] A. Kastner, C. Lux, T. Ring, S. Zullighoven, C. Sarpe, A. Senftleben, T. Baumert ChemPhysChem. 2016, 17, 1119-1122.

[13] J. Miles, D. Fernandes, A. Young, C. M. M. Bond, S. W. Crane, O. Ghafur, D. Townsend, J. Sá, J. B. Greenwood Analytica Chimica Acta. 2017, 984, 134-139.

[14] F. Partal Urena, J. R. Aviles Moreno, J. J. Lopez Gonzalez Tetrahedron-Asymmetry. 2009, 20, 8997.

[15] P. Brint, E. Meshulam, A. Gedanken Chem. Phys. Lett. 1984, 109, 383-387.

[16] G. A. Garcia, L. Nahon, I. Powis Int. J. Mass. Spec. 2003, 225, 261-270.

[17] M. A. Smialek, M. J. Hubin-Franskin, J. Delwiche, D. Duflot, N. J. Mason, S. Vronning-Hoffmann, G. G. B. de Souza, A. M. F. Rodrigues, F. N. Rodrigues, P. Limao-Vieira Phys. Chem. Chem. Phys. 2012, 14, 2056-2064.

[18] W. Z. Fang, L. Gong, X. B. Shan, Y. J. Zhao, F. Y. Liu, Z. Y. Wang, L. S. Sheng Journal of Mass Spectrometry. 2011, 46, 1152-1159.

[19] C. Loge, U. Boesl ChemPhysChem. 2011, 12, 1940-1947.

[20] M. M. Rafiee Fanood, M. H. M. Janssen, I. Powis J. Chem. Phys. 2016, 145, 124320.

[21] M. M. Rafiee Fanood, M. H. M. Janssen, I. Powis Phys. Chem. Chem. Phys. 2015, 17, 8614-8617. [22] S. Beaulieu, A. Ferre, R. Geneaux, R. Canonge, D. Descamps, B. Fabre, N. Fedorov, F. Legare, S. Petit, T. Ruchon, V. Blanchet, Y. Mairesse, B. Pons New J. Phys. 2016, 18, 102002.

[23] B. Ritchie Phys. Rev. A. 1976, 13, 1411-1415.

[24] L. Nahon, G. A. Garcia, I. Powis J. Elec. Spec. Rel. Phen. 2015, 204, 322-334.

[25] A. Comby, S. Beaulieu, M. Boggio-Pasqua, D. Descamps, F. Legare, L. Nahon, S. Petit, B. Pons, B. Fabre, Y. Mairesse, V. Blanchett J. Phys. Chem. Lett. 2016, 7, 4514-4519.

[26] D. Desiderio, S. D. Fonzo, B. Diviacco, W. Jark, J. Krempasky, R. Krempaska, F. Lama, M. Luce, H.C. Mertins, M. Piacentini, T. Prosperi, S. Rinaldi, G. Soullie, F.Schäfers, F. Schmolle, L. Stichauer, S. Turchini, R. P. Walker, N. Zema Synchrotron Radiation News. 1999, 12, 34.

[27] I. Powis in Photoelectron Circular Dichroism in Chiral Molecules, Vol. 138 (Ed. J. C. Light), Wiley, New York, 2008, pp.267-329. 
[28] K. N. Huang Phys. Rev. A. 1980, 22, 223-239.

[29] L. Nahon, N. d. Oliveira, G. Garcia, J. F. Gil, B. Pilette, O. Marcouille, B. Lagarde, F. Polack J.

Synchrot. Radiat. 2012, 19, 508-520.

[30] L. Nahon, C. Alcaraz Applied Optics. 2004, 43, 1024-1037.

[31] B. Mercier, M. Compin, C. Prevost, G. Bellec, R. Thissen, O. Dutuit, L. Nahon J. Vac. Sci. Tech. A. 2000, 18, 2533-2541.

[32] G. A. Garcia, B. K. C. de Miranda, M. Tia, S. Daly, L. Nahon Rev. Sci. Inst. 2013, 84, 053112.

[33] X. F. Tang, G. A. Garcia, J. F. Gil, L. Nahon Rev. Sci. Inst. 2015, 86, 123108.

[34] L. Nahon, G. A. Garcia, C. J. Harding, E. A. Mikajlo, I. Powis J. Chem. Phys. 2006, 125, 114309.

[35] G. A. Garcia, L. Nahon, I. Powis Rev. Sci. Inst. 2004, 75, 4989-4996.

[36] Personal communication luliia Myrgorodska and Uwe Meierhenrich.

[37] B. Sztaray, T. Baer Rev. Sci. Inst. 2003, 74, 3763-3768.

[38] G. A. Garcia, H. Soldi-Lose, L. Nahon Rev. Sci. Inst. 2009, 80, Art. no. 023102.

[39] J. C. Poully, J. P. Schermann, N. Nieuwjaer, F. Lecomte, G. Gregoire, C. Desfrancois, G. A. Garcia, L. Nahon, D. Nandi, L. Poisson, M. Hochlaf Phys. Chem. Chem. Phys. 2010, 12, 3566-3572.

[40] B. Jansik, A. Rizzo, L. Frediani, K. Ruud, S. Coriani J. Chem. Phys. 2006, 125, 234105.

[41] M. J. Frisch, G. W. Trucks, H. B. Schlegel, G. E. Scuseria, M. A. Robb, J. R. Cheeseman, G.

Scalmani, V. Barone, B. Mennucci, G. A. Petersson, H. Nakatsuji, M. Caricato, X. Li, H. P. Hratchian, A. F. Izmaylov, J. Bloino, G. Zheng, J. L. Sonnenberg, M. Hada, M. Ehara, K. Toyota, R. Fukuda, J.

Hasegawa, M. Ishida, T. Nakajima, Y. Honda, O. Kitao, H. Nakai, T. Vreven, J. J. A. Montgomery, J. E. Peralta, F. Ogliaro, M. Bearpark, J. J. Heyd, E. Brothers, K. N. Kudin, V. N. Staroverov, T. Keith, R. Kobayashi, J. Normand, K. Raghavachari, A. Rendell, J. C. Burant, S. S. Iyengar, J. Tomasi, M. Cossi, N. Rega, J. M. Millam, M. Klene, J. E. Knox, J. B. Cross, V. Bakken, C. Adamo, J. Jaramillo, R. Gomperts, R. E. Stratmann, O. Yazyev, A. J. Austin, R. Cammi, C. Pomelli, J. W. Ochterski, R. L. Martin, K. Morokuma, V. G. Zakrzewski, G. A. Voth, P. Salvador, J. J. Dannenberg, S. Dapprich, A. D. Daniels, O. Farkas, J. B. Foresman, J. V. Ortiz, J. Cioslowski, D. J. Fox in Gaussian 09 Revision D.01, Vol. (Ed.^Eds.: Editor), Gaussian Inc., City, 2013.

[42] W. von Niessen, J. Schirmer, L. S. Cederbaum Comp. Phys. Rep. 1984, 1, 57-125.

[43] V. G. Zakrzewski, J. V. Ortiz, J. A. Nichols, D. Heryadi, D. L. Yeager, J. T. Golab Int. J. Quantum. Chem. 1996, 60, 29-36.

[44] I. Pugliesi, K. Muller-Dethlefs J. Phys. Chem. A. 2006, 110, 4657-4667.

[45] J. R. A. Moreno, T. R. Huet, J. J. L. Gonzalez Structural Chemistry. 2013, 24, 1163-1170.

[46] P. Downie, I. Powis J. Chem. Phys. 1999, 111, 4535-4547.

[47] I. Powis J. Chem. Phys. 2000, 112, 301-310.

[48] M. Stener, D. D. Tommaso, G. Fronzoni, P. Decleva, I. Powis J. Chem. Phys. 2006, 124, 024326.

[49] G. A. Garcia, L. Nahon, C. J. Harding, I. Powis Phys. Chem. Chem. Phys. 2008, 10, 1628-1639.

[50] I. Powis, C. J. Harding, G. A. Garcia, L. Nahon ChemPhysChem. 2008, 9, 475-483.

[51] J. G. Norman J. Chem. Phys. 1974, 61, 4630-4635.

[52] G. A. Garcia, H. Dossmann, L. Nahon, S. Daly, I. Powis Phys. Chem. Chem. Phys. 2014, 16, 1621416224.

[53] S. Stranges, S. Turchini, M. Alagia, G. Alberti, G. Contini, P. Decleva, G. Fronzoni, M. Stener, N. Zema, T. Prosperi J. Chem. Phys. 2005, 122, 244303.

[54] M. Tia, B. Cunha de Miranda, S. Daly, F. Gaie-Levrel, G. A. Garcia, L. Nahon, I. Powis J. Phys. Chem. A. 2014, 118, 2765-2779. 
[55] S. Turchini, D. Catone, N. Zema, G. Contini, T. Prosperi, P. Decleva, M. Stener, F. Rondino, S. Piccirillo, K. C. Prince, M. Speranza ChemPhysChem. 2013, 14, 1723-1732.

[56] S. Turchini, D. Catone, G. Contini, N. Zema, S. Irrera, M. Stener, D. Di Tommaso, P. Decleva, T. Prosperi ChemPhysChem. 2009, 10, 1839-1846.

[57] D. Di Tommaso, M. Stener, G. Fronzoni, P. Decleva ChemPhysChem. 2006, 7, 924-934.

[58] C. J. Harding, I. Powis J. Chem. Phys. 2006, 125, 234306.

[59] A. Hong, C. M. Choi, H. J. Eun, C. Jeong, J. Heo, N. J. Kim Angew. Chem.-Int. Edit. 2014, 53, 78057808. 


\section{Tables}

Table 1 Calculated Conformer Structures for S-limonene ${ }^{a}$

\begin{tabular}{|c|c|c|c|c|c|c|}
\hline & \multicolumn{6}{|c|}{ Dihedral Angle $\angle a b c d$} \\
\hline & Eq-1 & Eq-1 $x^{+}$ & $\mathrm{Eq}-2$ & Eq-2 $x^{+}$ & Eq-3 & Eq-3 $X^{+}$ \\
\hline CASSCF/cc-pVDZ & -107.3 & -107.7 & 106.8 & 105.2 & 31.9 & 33.4 \\
\hline MP2/cc-pVTZ & -108.4 & -109.6 & 112.4 & 111.2 & 19.4 & 11.6 \\
\hline MP2/cc-pVDZ & -108.2 & -108.7 & 111.2 & 110.0 & 23.8 & 14.4 \\
\hline B3LYP/cc-pVDZ & -109.4 & -105.1 & 111.6 & 69.8 & 22.1 & 17.4 \\
\hline M11(UF)/cc-pVDZ & -104.1 & -105.8 & 117.4 & 116.3 & 0.4 & 1.4 \\
\hline MP2/cc-pVDZ ${ }^{b, c, d}$ & -108.5 & -89.5 & 112.5 & 109.9 & 20.9 & 38.3 \\
\hline B3LYP/cc-pVDZ d,e & -109.3 & & 112.7 & & 22 & \\
\hline MP2/6-31G**d,f & -107 & & 113 & & 21 & \\
\hline & & & & & & \\
\hline
\end{tabular}

${ }^{a}$ Additional calculation results are presented as Supporting Information

${ }^{\mathrm{b}}$ Measured from the Cartesian structures provided as Electronic Supporting Information to Ref. [17]

c Ion calculations used MP2-ZAPT method

d Original values were obtained for the R-enantiomer. These have been converted to the corresponding S-enantiomer value by negation.

e Ref. [14]

${ }^{f}$ Ref. [45] 
Table 2 Calculated Conformer Energies ${ }^{\mathrm{a}}$ and Boltzmann Populations (300K)

\begin{tabular}{|l|l|l|l|l|l|l|l|l|}
\hline & $\Delta \mathrm{E}^{\mathrm{a}, \mathrm{b}}$ & \% Pop. $^{\mathrm{b}}$ & $\Delta \mathrm{E}^{\mathrm{a}, \mathrm{c}}$ & \% Pop. $^{\mathrm{c}}$ & $\Delta \mathrm{E}^{\mathrm{a}, \mathrm{d}}$ & \% Pop. $^{\mathrm{d}}$ & $\Delta \mathrm{E}^{\mathrm{a}, \mathrm{e}}$ & Pop. $^{\mathrm{e}}$ \\
\hline Eq-1 & 0.0 & 42.8 & 0.0 & 51 & 0.0 & 55 & 0.0 & 48 \\
\hline Eq-2 & 0.69 & 32.4 & 1.2 & 32 & 1.13 & 29 & 0.96 & 33 \\
\hline Eq-3 & 1.71 & 21.4 & 2.8 & 17 & 2.80 & 16 & 2.37 & 19 \\
\hline & & & & & & & & \\
\hline Ax-1 & 13.41 & 0.2 & & & & & & \\
\hline Ax-2 & 14.58 & 0.1 & & & & & & \\
\hline Ax-3 & 6.49 & 3.1 & & & & & & \\
\hline
\end{tabular}

${ }^{\mathrm{a}} \mathrm{kJ} \mathrm{mol}^{-1}$ with ZPE corrections applied.

${ }^{b} \operatorname{Ref}[14]$ B3LYP/cc-pVDZ

${ }^{c} \operatorname{Ref}$ [45] MP2/6-311++G(2df,p)

${ }^{d}$ Ref [17] CCSD/cc-pVDZ

${ }^{\text {e }}$ Current work MP2/cc-pVTZ. Additional results are presented in Supporting Information. 
Table 3 Calculated ${ }^{\mathrm{a}} \mathrm{X}$-band ionization energies (eV)

\begin{tabular}{|c|c|c|c|c|c|c|}
\hline \multirow[b]{2}{*}{ Vertical I.E.s } & \multicolumn{3}{|c|}{ HOMO $2 \pi^{-1}$} & \multicolumn{3}{|c|}{ HOMO-1 $1 \pi^{-1}$} \\
\hline & Eq-1 & Eq-2 & Eq-3 & Eq-1 & Eq-2 & Eq-3 \\
\hline OVGF/6-311G**//MP2/cc-pVTZ & 8.534 & 8.575 & 8.538 & 8.962 & 8.977 & 8.993 \\
\hline OVGF/cc-pVDZ//MP2/cc-pVTZ & 8.619 & 8.657 & 8.619 & 9.159 & 9.169 & 9.088 \\
\hline P3/cc-pVDZ//MP2/cc-pVDZ ${ }^{b}$ & 8.472 & 8.508 & 8.471 & 8.897 & 8.899 & 8.912 \\
\hline RCCSD(T)/cc-pVDZ//MP2/ccpVDZ ${ }^{b}$ & 8.591 & 8.610 & 8.586 & & & \\
\hline Adiabatic I.E.s & & & & & & \\
\hline MP2/cc-pVDZ ${ }^{c}$ & 8.402 & 8.443 & 8.453 & & & \\
\hline RCCSD(T)/cc-pVDZ//MP2/ccpVDZ ${ }^{b}$ & 8.558 & 8.279 & 8.579 & & & \\
\hline
\end{tabular}

${ }^{a}$ Additional orbitals calculated in the current work are listed in Supporting Information

${ }^{b}$ Ref. [17]

${ }^{c} \Delta \mathrm{E}_{0+Z \mathrm{ZPE}}$ calculation. Results using other methods and basis sets are listed in Supporting Information. 
Figures
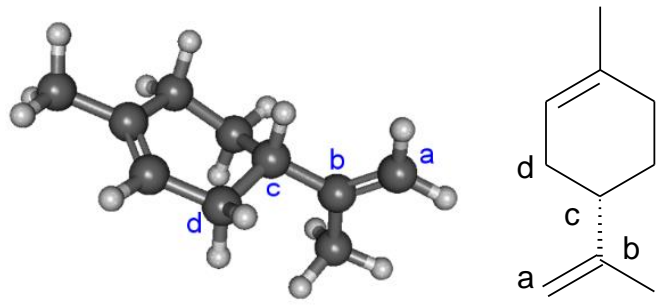

Figure 1. S-limonene in its most stable equatorial conformation, Eq-1. The conformers can be distinguished by the dihedral angle $a-b-c-d$. Atom $c$ is the chiral centre. 


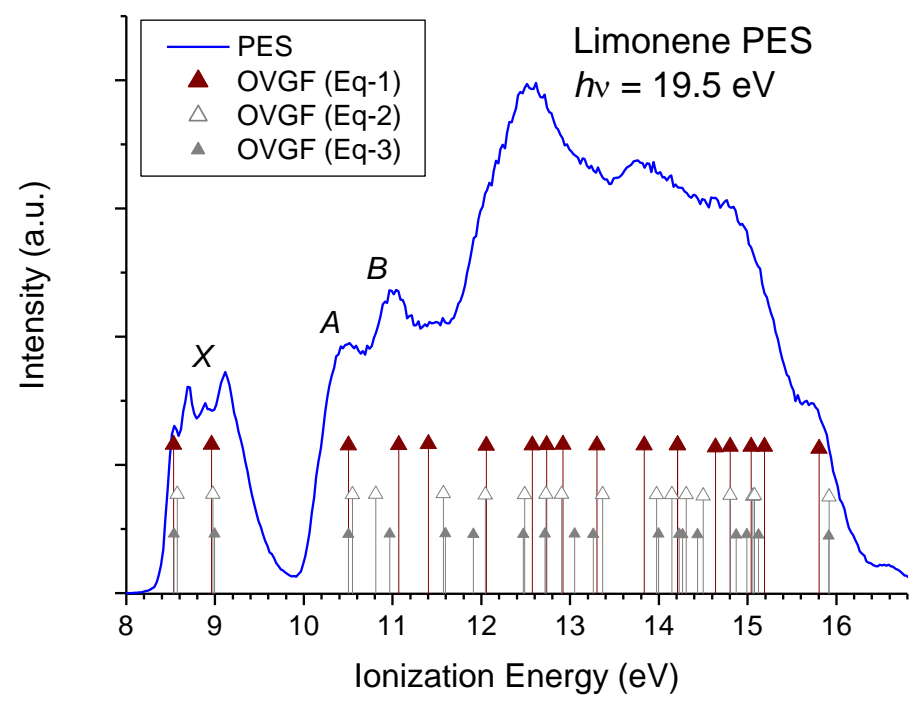

Figure 2. Outer valence region photoelectron spectrum of limonene recorded at Elettra. Also marked on the plot are the calculated OVGF/6-311G** orbital ionization energies for the three equatorial conformers (see text) of limonene. 


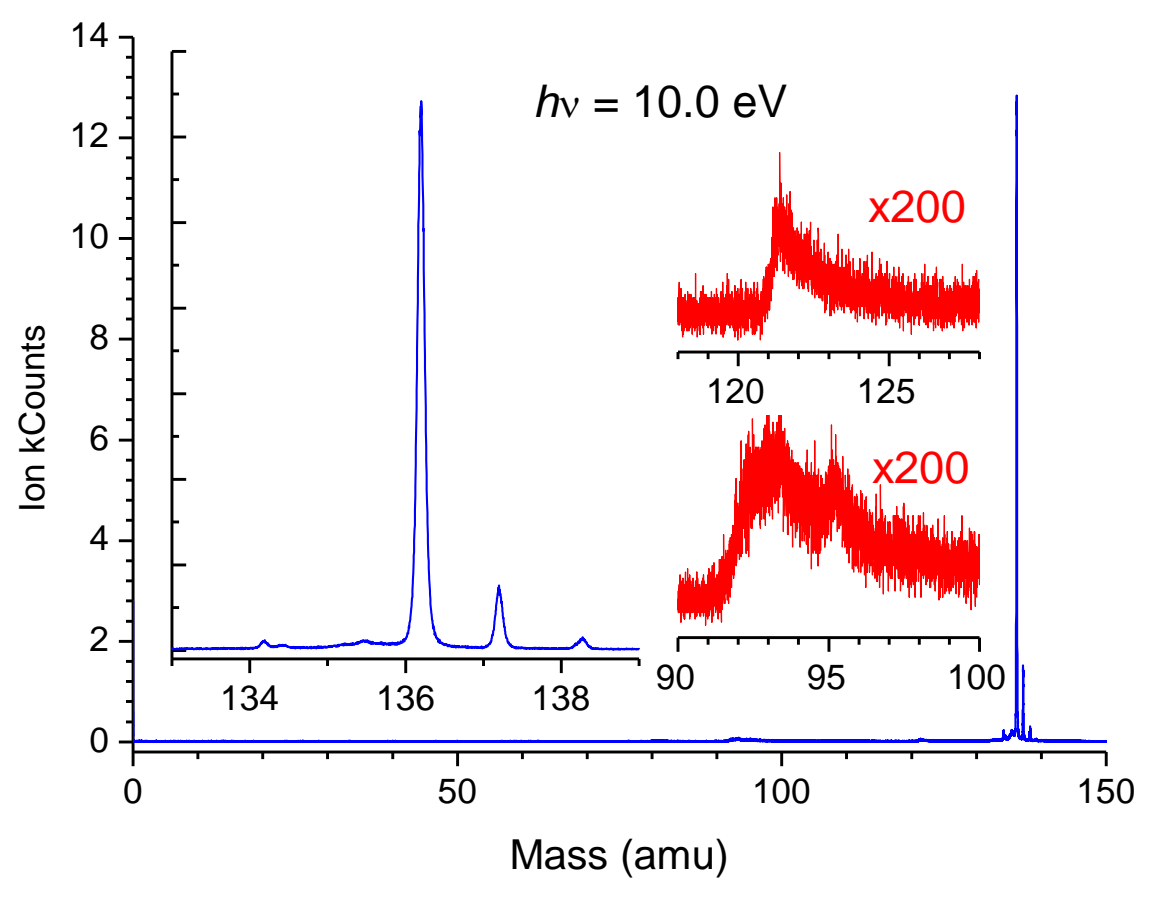

Figure 3 lon time-of-flight mass spectrum of limonene recorded at $h v=10.0 \mathrm{eV}$ in a cold molecular beam. The left inset shows the parent ion $(\mathrm{m} / \mathrm{z}=136.2)$ region with an expanded mass scale; the centre insets shows the 90-100 amu and 118-128 amu regions with a $\times 200$ expansion of the vertical intensity scale. 


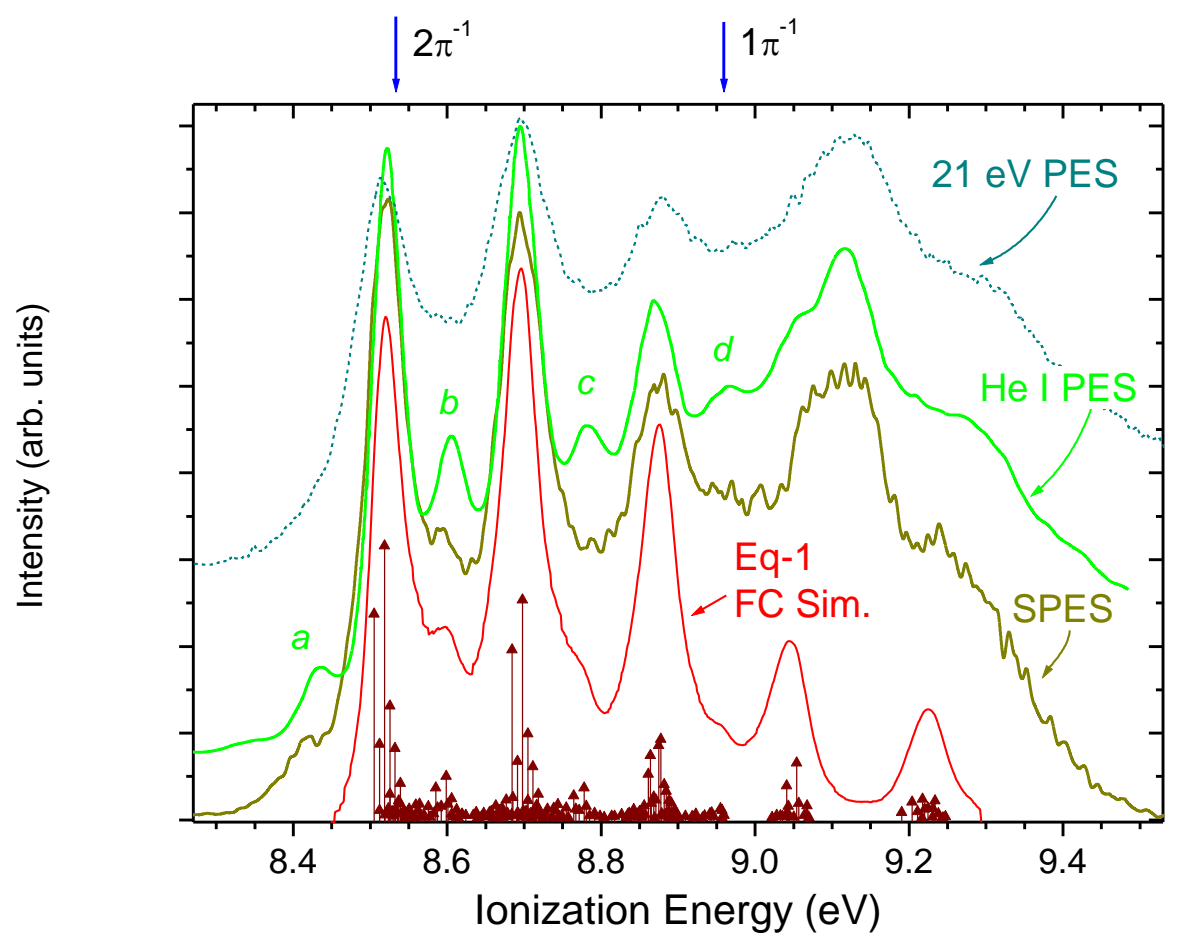

Fig. 4 Slow photoelectron spectrum (SPES) of the first band of jet cooled limonene, compared with the room temperature He I PES (Ref [17] and a Franck-Condon simulation of the cold Eq-1 conformer HOMO $2 \pi^{-1}$ ionization. For clarity small vertical displacements have been applied to the baselines of these spectra. A lower resolution PES ( $h v=21 \mathrm{eV}$, pass energy $1 \mathrm{eV}, 100 \mu \mathrm{m}$ slits) recorded with an effusive room temperature source at Elettra is also shown. The simulation uses MP2/cc-pVDZ calculated harmonic vibrational frequencies for the Eq-1 conformer, uniformly scaled by a factor of 0.91 . The 200 most intense individual transitions are plotted in the stick spectrum, while the complete transition set has been folded with a shaping function (FWHM $30 \mathrm{meV}$ ) chosen to provide the more realistic simulated vibrational profile shown. Also indicated at the top of the figure are the positions of the OVGF/6-311G(d,p) calculated $2 \pi^{-1}$ and $1 \pi^{-1}$ vertical ionization energies. 

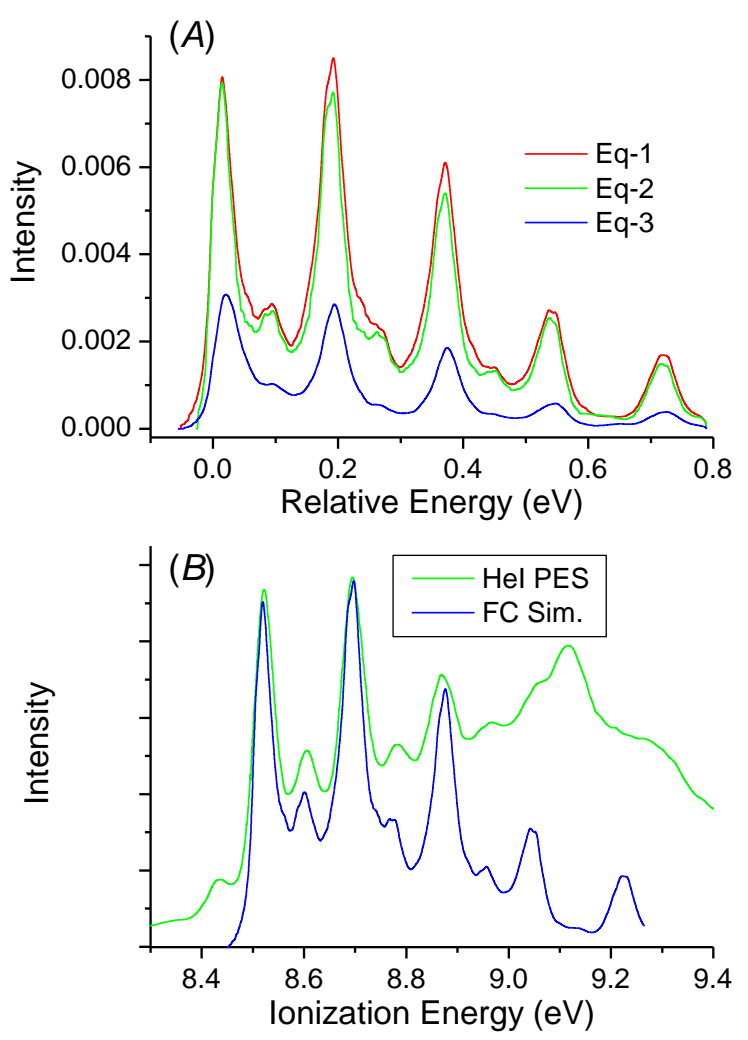

Fig. 5 (A) A comparison of MP2/cc-pVDZ FC simulations for the three conformers Eq-1, Eq-2, Eq-3. The calculated stick spectra have been convoluted with Lorentzian functions of FWHM 20 meV for plotting. (B) 300K 5:3:2 Boltzmann population weighted combination of Eq-1:Eq-2:Eq3 simulations with Eq-3 origin offset by $+0.81 \mathrm{meV}$ (see text). Also included is the He I spectrum from Ref[17]. 


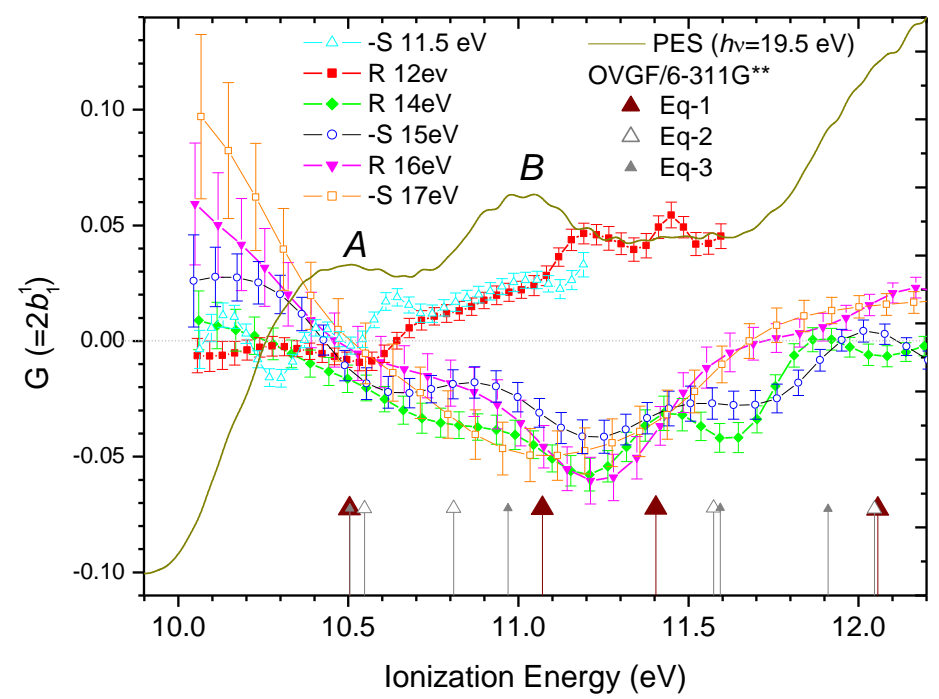

Fig. 6 VMI-PECD measurements of the $A / B$ band regions ( $3 r d, 4^{\text {th }}, \ldots$ orbital ionizations) of jet-cooled limonene at various photon energies. The figure includes measurements made with both $\mathrm{R}$ - and $\mathrm{S}$ enantiomers. PECD of the latter have been negated for plotting, reflecting the anticipated mirroring displayed by two enantiomers; this presentation facilitates direct visual comparison of the results. For reference the corresponding region of the full PES (Fig. 2) is plotted on the same energy scale. The photon energy at which each individual measurements was made is indicated in the plot key. 


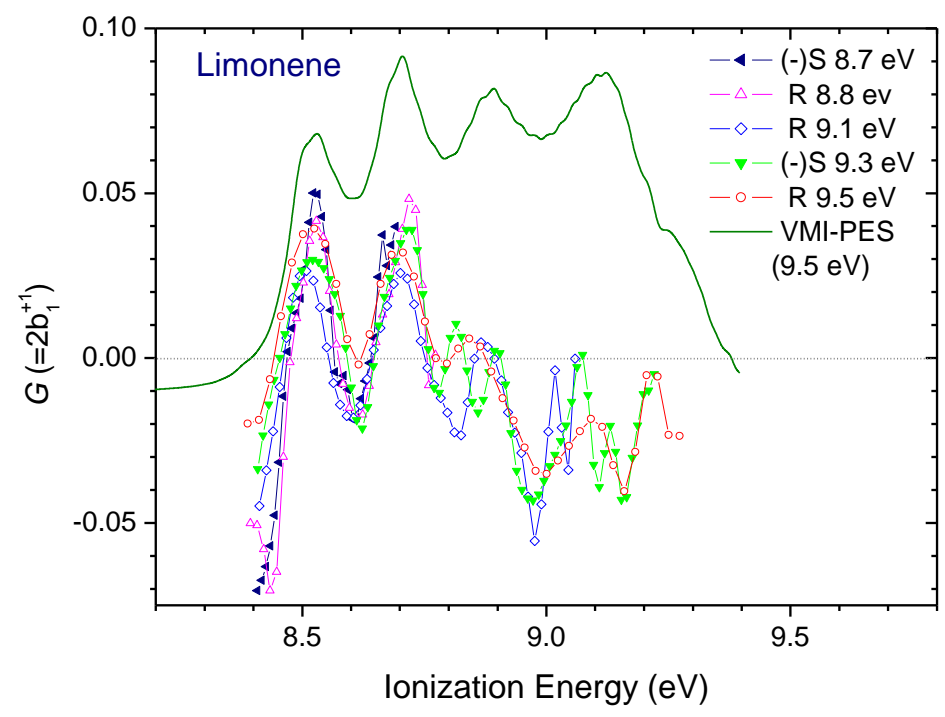

Fig. $7 \mathrm{VMI-PECD}$ results for limonene recorded at photon energies $8.7 \mathrm{eV}-9.5 \mathrm{eV}$. The figure includes $X$-band measurements made with both $\mathrm{R}$ - and $\mathrm{S}$ - enantiomers. PECD of the latter have been negated for plotting, as in Fig. 6 . The VMI-PES recorded with the $h v=9.5 \mathrm{eV}$ measurements is shown on the common energy scale. 


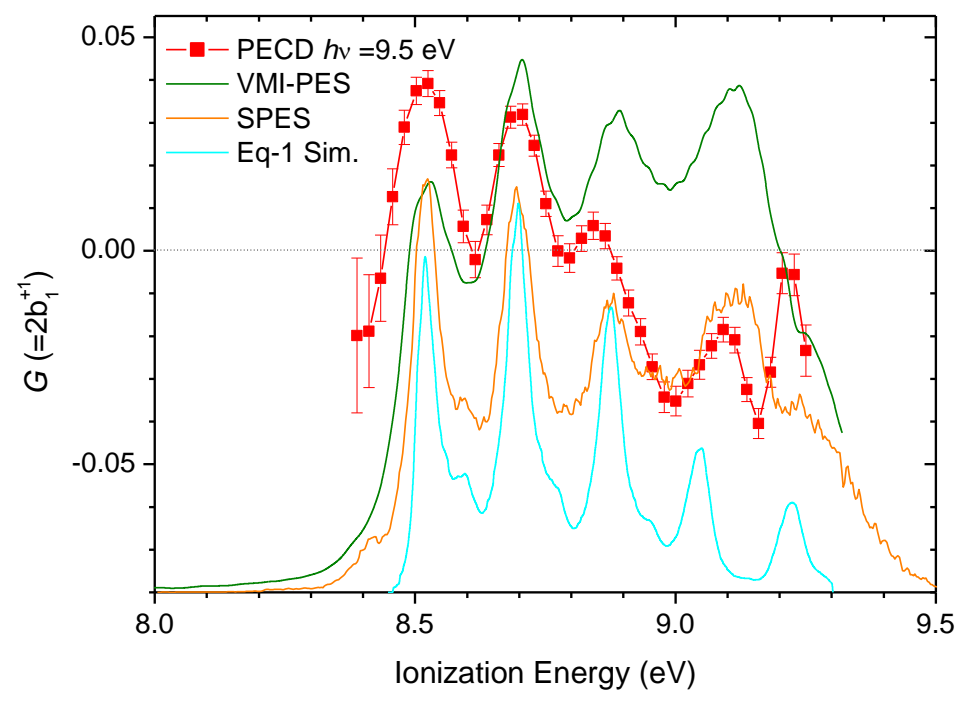

Fig 8. VMI-PECD for jet cooled R-limonene at $h v=9.5 E V$. The VMI-PES extracted from the combined $R C P+L C P$ set of electron images is plotted on the same energy scale. For additional reference so also are the SPES and an Eq-1 $2 \pi^{-1} \mathrm{FC}$ simulation of the PES using scaled M11/cc-pVDZ harmonic frequencies. The VMI-PES, SPES, and FC simulation all have arbitrarily normalised intensities. 


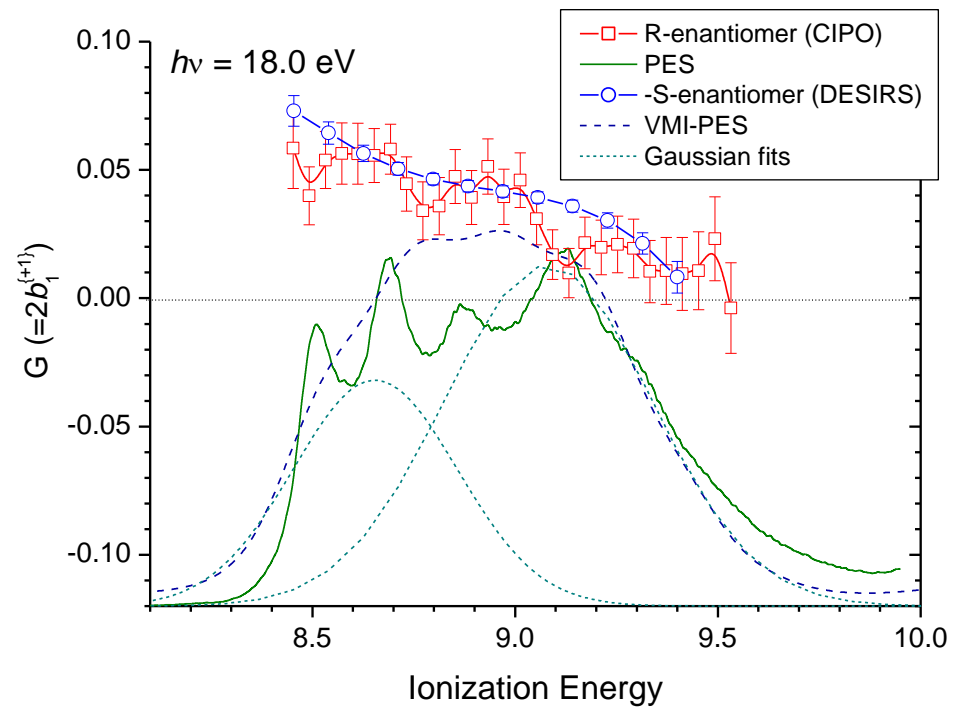

Fig $9 X$ band PES and PECD recorded with R-limonene dispersive energy analysis on the CIPO beamline at $h v=18 \mathrm{eV}$, plotted on a common ionization energy scale. For comparison the corresponding VMI-PES and PECD recorded at the same photon energy with S-limonene (but with PECD negated for plotting and visual comparison) are over-plotted. Both PES and VMI-PES are arbitrarily scaled. The figure also shows two Gaussian functions that fit to the VMI-PES profile. 


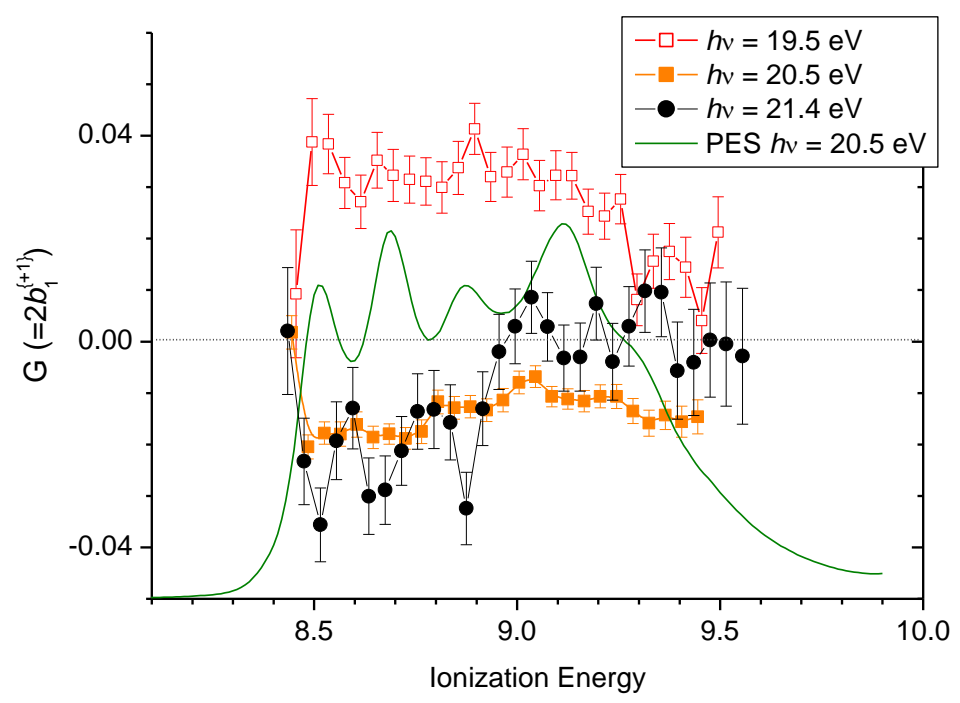

Fig 10 R-limonene X-band PECD recorded with dispersive energy analysis (CIPO beamline) at $h v=$ $19.5 \mathrm{eV}, 20.5 \mathrm{eV}, 21.4 \mathrm{eV}$. For reference, an example PES, simultaneously recorded under the same conditions, is shown with an arbitrary intensity scaling. 


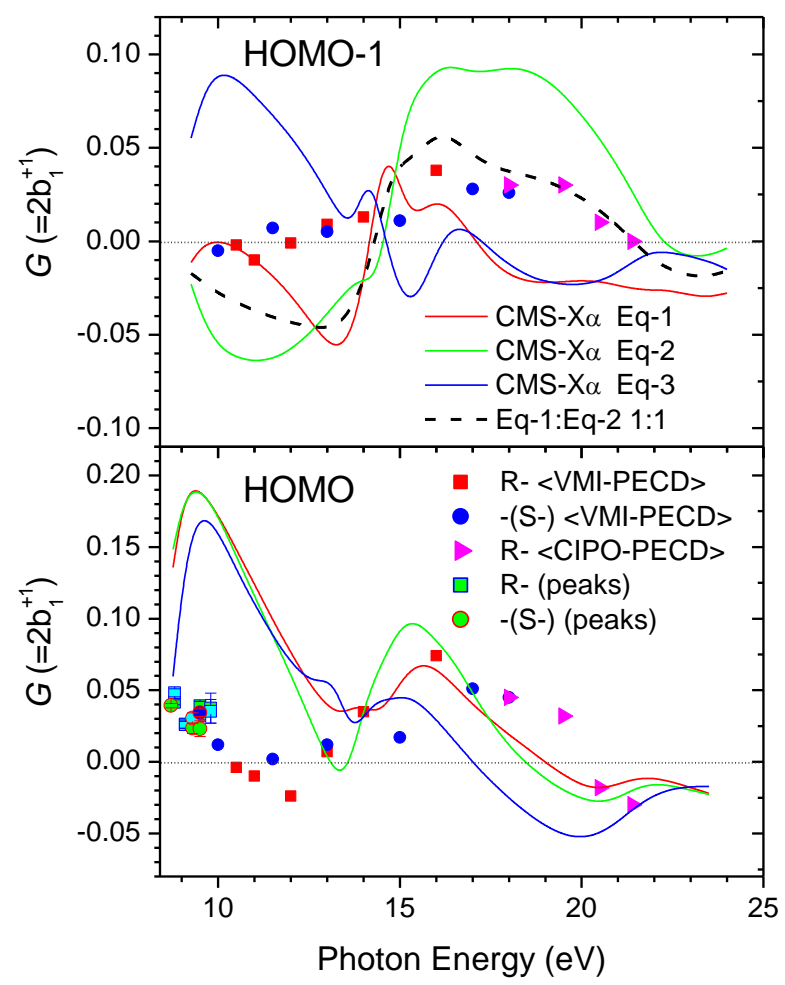

Fig. 11 Estimated mean PECD values for the HOMO $2 \pi^{-1}$ and HOMO-1 $1 \pi^{-1}$ ionizations in limonene, plotted as a function of the ionizing photon energy (see text for detail of how these are extracted from the experimental data). Calculated PECD curves obtained for the three equatorial conformers of limonene are shown for comparison. For plotting these theoretically predicted magnitudes have been scaled by a factor $50 \%$. For the HOMO-1 a 1:1 weighted average of the Eq-1:Eq-2 conformers, i.e. excluding the Eq-3 conformer is also shown. 
Photoionization of limonene enantiomers by circularly polarized synchrotron light produces a forward-backward asymmetry in the photoelectron angular distribution. This asymmetry is found to strongly modulate, even reversing in direction, with vibrational excitation observed in the limonene

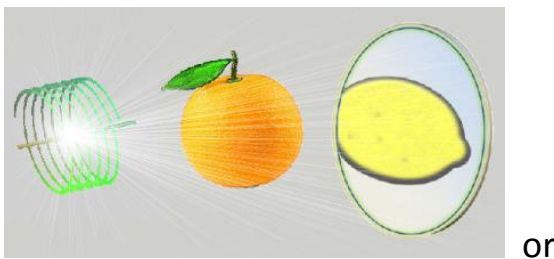

